\title{
BMJ Open Herpes zoster related healthcare burden and costs in immunocompromised (IC) and IC-free populations in England: an observational retrospective database analysis
}

\author{
Desmond Curran, ${ }^{\oplus}$ Manjit Hunjan, ${ }^{2}$ Amale El Ghachi, ${ }^{3}$ Yassine El-Hahi, ${ }^{4}$ \\ Veronique Bianco, ${ }^{5}$ Germano Ferreira ${ }^{6}$
}

To cite: Curran D, Hunjan M, El Ghachi A, et al. Herpes zoster related healthcare burden and costs in immunocompromised (IC) and IC-free populations in England: an observational retrospective database analysis. BMJ Open 2019;9:e023502. doi:10.1136/ bmjopen-2018-023502

- Prepublication history and additional material for this paper are available online. To view please visit the journal (http:// dx.doi.org/10.1136/bmjopen2018-023502).

Received 9 April 2018 Revised 24 June 2019 Accepted 2 July 2019

Check for updates

(c) Author(s) (or their employer(s)) 2019. Re-use permitted under CC BY-NC. No commercial re-use. See rights and permissions. Published by BMJ.

${ }^{1}$ GSK, Wavre, Belgium ${ }^{2}$ GSK, Uxbridge, United Kingdom ${ }^{3}$ Aixial, Boulogne-Billancourt, France

${ }^{4}$ Valesta, Mechelen, Belgium

${ }^{5}$ GSK, Rockville, Maryland, USA

${ }^{6} \mathrm{P}-95$ Epidemiology and

pharmacovigilance services, Heverlee, Belgium

Correspondence to Dr Desmond Curran; desmond.x.curran@gsk.com

\section{ABSTRACT}

Objective Individuals with immunocompromised (IC) conditions are at a higher risk of developing herpes zoster $(\mathrm{HZ})$ than IC-free individuals. This study assessed the healthcare resource utilisation (HCRU) burden and costs, of $\mathrm{HZ}$ in IC and IC-free individuals $\geq 18$ years of age (YOA). Methods We conducted an observational retrospective study in a cohort of IC $(n=621588)$ and IC-free $(n=621588)$ individuals, matched by age, gender and General Practitioner practice region, contributing to the Clinical Practice Research Datalink database from 2000 to 2012 and linked to the Hospital Episode Statistics inpatient data. HCRU (ie, primary and secondary care consultations, hospital inpatient stays and treatment prescriptions) was analysed from 7 days before to: (1) 30, (2) 365 days after the $\mathrm{HZ}$ diagnosis date for individuals with (1) HZ only (no postherpetic neuralgia (PHN)) and (2) individuals with $\mathrm{HZ}$ and PHN only. Healthcare costs were computed by multiplying the number of units of resources used by the unit costs, summed across all HCRU categories to obtain a total cost per subject. Values were expressed in 2014 UK pound sterling ( $£$ ) and presented for $\mathrm{HZ}$ cases overall, stratified by age (ie, 18-49, 50-59, 60-69, 70-79 and $\geq 80$ YOA) and IC status

Results The percentage of $\mathrm{HZ}$ cases requiring hospitalisation was higher in IC individuals $(2.7 \%$ vs $0.4 \%$ in IC and IC-free individuals aged 18-49 YOA, respectively and $9.5 \%$ vs $7.5 \%$ in IC and IC-free individuals aged $\geq 80$ YOA, respectively). Similarly, HZ-related mean treatment costs per subject were higher in IC individuals ( $£ 189$ vs £104 in IC and IC-free individuals aged 18-49 YOA, respectively and $£ 557$ vs $£ 401$ in IC and IC-free individuals aged $\geq 80$ YOA, respectively). Costs varied considerably by IC condition.

Conclusions Individuals with IC conditions, have a high burden of $\mathrm{HZ}$, associated with an increased risk of $\mathrm{HZ}$ and high HZ-related healthcare costs.

\section{INTRODUCTION}

Varicella zoster virus cell-mediated immunity (VZV-CMI) inhibits the development of herpes zoster (HZ). ${ }^{1}$ Therefore, if for any

\section{Strengths and limitations of this study}

- The study is an observational retrospective descriptive study presenting the healthcare resource utilisation and costs associated with herpes zoster $(\mathrm{HZ})$ in both immunocompromised (IC) and IC-free populations aged $\geq 18$ years of age in England.

- The IC population included 621588 individuals who were registered in the Clinical Practice Research Datalink from January 2000 to March 2012 with $\geq 12$ month follow-up before being diagnosed with any of the selected 16 IC conditions and matched to the Hospital Episode Statistics database by age, gender and practice location to extract the IC-free population $(n=621588)$.

- The particularity of this study is that the design allowed calculation of IC condition prevalence rates, $\mathrm{HZ}$ incidence rates and occurrence of $\mathrm{HZ}$-related healthcare utilisation and costs at individual level in the same predefined population(s).

- This key study will provide data to be used in economic analyses to evaluate the value of vaccination in reducing the burden of $\mathrm{HZ}$ in $\mathrm{IC}$ populations.

- A limitation of the study is that the diagnoses were derived from administrative codes, which are recognised to be subject to miscoding or under-coding and are not validated against medical charts.

reason VZV-CMI declines, the risk of $\mathrm{HZ}$ increases. Reasons for VZV-CMI decline can include, increasing age and immune suppression. VZV-CMI is not optimal in individuals with immunocompromised (IC) conditions and the age-specific incidence and severity of $\mathrm{HZ}$ greatly increases in IC patients due to underlying illness (eg, Human immunodeficiency virus infection) or immunosuppressive therapies for autoimmune disease, malignancy, or organ transplantation. ${ }^{2}$

The incidence and severity of $\mathrm{HZ}$ is marked with an increase in people $\geq 50$ years of age 
(YOA) due to an age-related decline in immunity (ARDI). In the United Kingdom (UK) the incidence of $\mathrm{HZ}$ rises from 7.1 per 1000 person-years (PY) among 60-64year olds to 12.2 per 1000 PY among individuals aged $\geq 85$ YOA. ${ }^{3}$ Further to the impact of ARDI, a study by Forbes $e t$ $a l$ in 2014 investigated the increased risk for HZ in the UK population, associated with autoimmune conditions such as rheumatoid arthritis, systemic lupus erythematosus; and chronic conditions such as diabetes mellitus, chronic obstructive pulmonary disease, chronic kidney disease and asthma. ${ }^{2}$ In addition to the increased risk of $\mathrm{HZ}$ in the various IC conditions these populations also experience increased severity of disease. In a study in Canada, Drolet et al reported that individuals with an impaired immune status had HZ severity of illness scores, as measured by the Zoster Brief Pain Inventory, which were twice as high as individuals with normal immune function. ${ }^{45}$ In a study in the USA, Yawn et al reported that although $8 \%$ of $\mathrm{HZ}$ cases were in individuals who were immunocompromised, these individuals represented $23.8 \%$ of the total HZ-related costs. ${ }^{6}$ The increase in healthcare costs was associated with higher rates of postherpetic neuralgia (PHN) and non-pain complications in this group of individuals. ${ }^{6}$

This study aims to estimate the healthcare resource utilisation of $\mathrm{HZ}$ in selected IC populations and in an IC-free (ie, immunocompetent) population aged $\geq 18 \mathrm{YOA}$ in England. The clinical burden of disease epidemiological results of the study are reported elsewhere, ${ }^{7}$ and may be summarised as follows: the prevalence of IC conditions increased from $7.6 \%$ in individuals aged $18-44$ YOA to $42.2 \%$ in individuals aged $\geq 80 \mathrm{YOA}$; the incidence rate of $\mathrm{HZ}$ in the IC cohort was $3.5 / 1000 \mathrm{PY}$ in individuals aged 18-49 YOA increasing to $12.6 / 1000$ PY in individuals aged $\geq 80 \mathrm{YOA}$. In this manuscript, we focus on the healthcare resource utilisation and costs associated with $\mathrm{HZ}$ in both IC and IC-free populations.

\section{METHODS}

The study was conducted as an observational retrospective descriptive study (e-track number: 201615), in a cohort of eligible matched IC and IC-free populations (aged $\geq 18 \mathrm{YOA}$ ). The IC population included individuals who were registered in the Clinical Practice Research Datalink (CPRD) from January 2000 to March 2012 with $\geq 12$ month follow-up before being diagnosed with any of the selected 16 IC conditions (see online supplementary material). The CPRD IC population cohort was linked to the Hospital Episode Statistics (HES) database and matched, using a 1:1 ratio, to a cohort of CPRD-HES linked IC-free population ( $\mathrm{n}=621588)$, by age, gender and practice location. ${ }^{8}$ Individuals with a missing date of IC diagnosis were excluded from the study population. Clinical diagnoses were based on READ codes used in CPRD and with the International Classifications of Diseases-10 ${ }^{\text {th }}$ revision (ICD-10) codes in the HES database.

The study protocol was approved by the Independent Scientific Advisory Committee (ISAC) for the Medicines and Healthcare Products Regulatory Agency database research (ISAC protocol number 14_222R). The study was conducted in accordance with all applicable regulatory requirements, with the Guidelines for Good Pharmacoepidemiology Practices, ${ }^{9}$ all applicable patient privacy requirements and the guiding principles of the Declaration of Helsinki.

The matched IC and IC-free cohorts were followed up from the index date until the earliest of the following events: transfer out of the practice date, the last GP practice collections date, death date or the end of the study. ${ }^{7}$ Healthcare resource data associated with an incident HZ episode during the study follow-up were extracted for IC and matched IC-free CPRD-HES-linked individuals. Only reported records (resource utilisation) with available event dates during the individuals' eligibility period

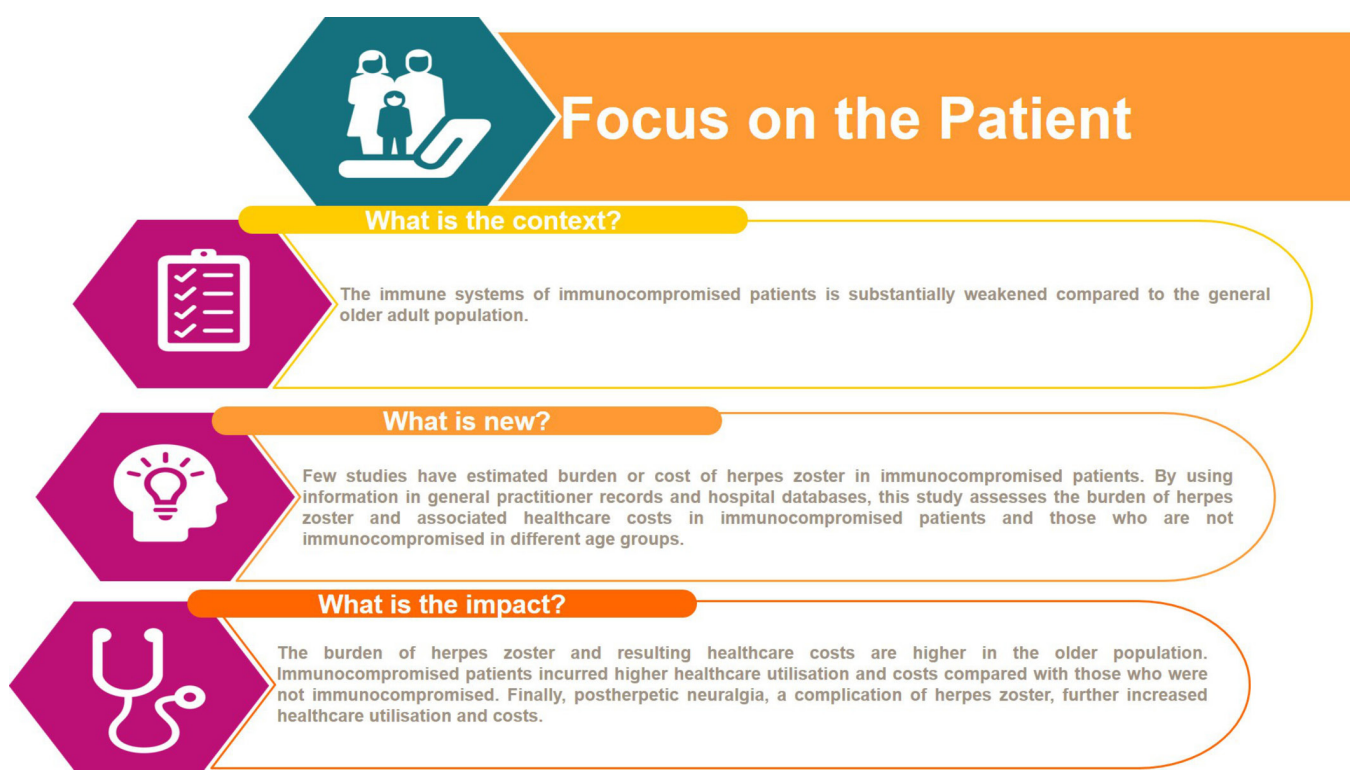

Figure 1 Lay language summary. 


\section{Panel A}

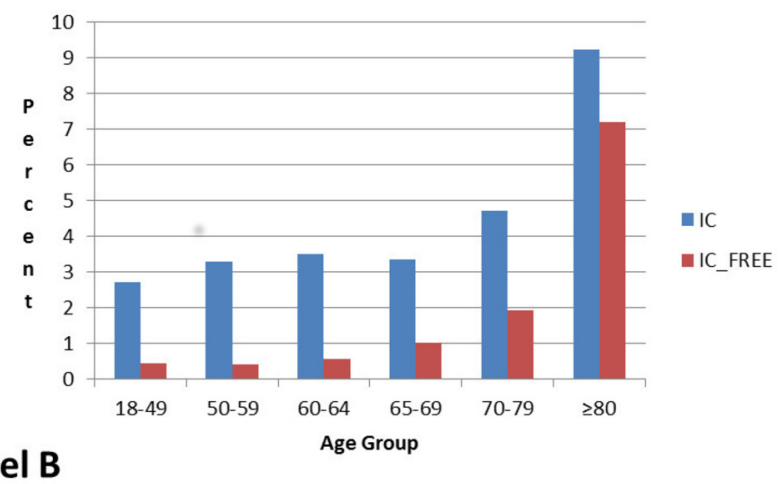

Panel B

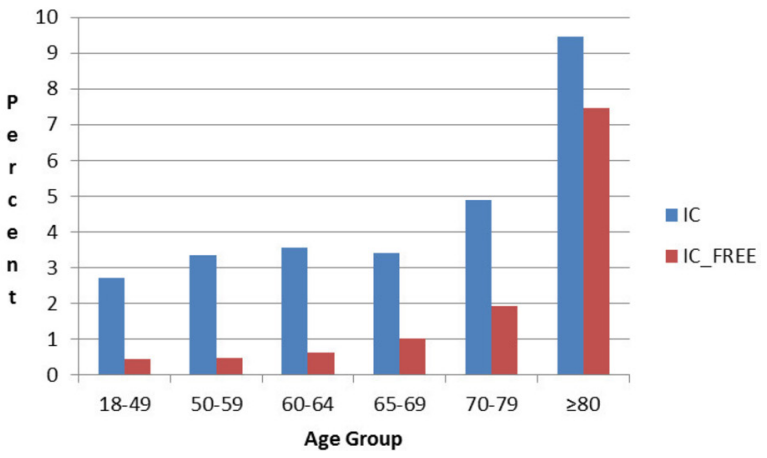

Figure 2 Inpatient hospital admission by HES-linked matched IC or IC-free cohort over the time periods: 7 days prior to 90 days post initial $\mathrm{HZ}$ onset (Panel A) and 7 days prior to 365 days post initial $\mathrm{HZ}$ onset (Panel B). For HZ individuals without PHN: data from 7 days prior to 30 days post $\mathrm{HZ}$ onset included. For $\mathrm{HZ}$ individuals with $\mathrm{PHN}$ : data from 7 days prior until the following time periods after $\mathrm{HZ}$ onset included-90 days (Panel A) and 365 days (Panel B). HES, Hospital Episode Statistics; HZ, herpes zoster; IC, immunocompromised; PHN, postherpetic neuralgia.

and those that occurred 7 days before the initial $\mathrm{HZ}$ onset date, up to 365 days after the initial HZ onset date, were extracted. Consequently, individuals who recorded the first PHN event date after 365 days post $\mathrm{HZ}$ event date were classified as not having PHN.

\section{Patient and public involvement}

This is a retrospective database analysis carried out following ethical committee approval. No patient or the public was involved in the study design or in the recruitment or the conduct of this study. No specific dissemination of study results to participants was done. However, we provided a lay language summary contextualising the results and potential clinical research relevance and impact in figure 1.

\section{Data sources}

Data were extracted from the following sources: (1) CPRD GOLD 2014Q3: Consultation, Clinical, Therapy and Referral datasets; (2) HES Inpatient 2013Q3: HES_DIAGNOSIS_EPI dataset; (3) HES Outpatient data (Set 9): Appointment and clinical datasets. Healthcare resource utilisation was defined as: HZ-treatment related prescribed medications; Consultations and care provided by General
Practitioners (GPs) or others in the GP practice); HES secondary care outpatient visits (HES outpatient events); and HES inpatient hospitalizations (HES inpatient events).

For each patient, healthcare costs stratified by subcategory of interest (HES Inpatient Hospitalizations; HES Outpatient consultations/visits; CPRD Ambulatory Visits; CPRD Other Ambulatory Visits; CPRD Prescriptions) were computed by multiplying units of resource use by their unit costs. These were then summed over all resource use categories to obtain a total cost for each patient. Values were expressed in $2014 \mathrm{UK}$ pound sterling (£).

\section{Healthcare resource costs}

For each patient, the cost of each prescription was calculated by merging the product code, package type and prescribed quantity with the associated standard package size and unit cost. The unit cost of a product in a prescription instance (ie, one distinct record in the CPRD therapy) was calculated using the cost described in the British National Formulary (BNF), 2015 (as listed price if included or indicative price based on price in $\mathrm{BNF}$ ).

Ambulatory visits included consultations with GPs and nurses in primary or community care. Visits included consultations at the practice or at the home of the patient, during working hours and out of hours. Consultations for which no clinical intervention was recorded were not included in the cost estimate for GP practice related healthcare utilisation, for example: information technology data migration, administrative recording of received information. Administrative resource use in primary care was considered, including time on the phone, writing reports, referrals, etc. A referral to secondary care noted in a patient's record, per se, was not allocated the cost of the secondary care appointment. The most conservative option for the cost per unit as included in the Personal Social Services Research Unit (PSSRU) Costs of Health and Social Care, 2014 were applied eg, GP consultation costs excluded qualification, direct staff care and travel costs. ${ }^{10}$ Where specific costs for 2013/14 were not available, 2012/13 costs, were adjusted by applying the Hospital and Community Health Services inflation index. ${ }^{10}$ Administration costs were based on unit costs as stated in the PSSRU, 2014.

Inpatient hospitalizations related to $\mathrm{HZ}$ were derived from HES data. Hospital Outpatient resource utilisation concerned HZ related referrals for non-inpatient hospital consultations, derived from the HES Outpatient data. Additionally, visits to the Accident and Emergency (A\&E) department in hospitals were also recorded and costed. Inpatient hospitalisation costs were based on the average cost per episode using HES data for 2013/14 (calculated from the total average payment by result spell cost and the average number of episodes per spell). Hospital outpatient costs were sourced from National Tariff costs (2014) for specific consultant led outpatient consultations; conservative costs were allocated that is wherever applicable costs for first attendance by a single professional appointment were used. ${ }^{11}$ Costs allocated to A\&E visits were based on the cost of a category 3 investigation with category $1-3$ treatment. ${ }^{11}$ 
Open access

Table 1 Mean number of healthcare resources by IC status, age group and analysis period

\begin{tabular}{|c|c|c|c|c|}
\hline & \multicolumn{2}{|c|}{ IC cohort } & \multicolumn{2}{|c|}{ IC-free cohort } \\
\hline & 90 day & 365 day & 90 day & 365 day \\
\hline \multicolumn{5}{|c|}{ HES Hospital admission } \\
\hline $18-49$ & 0.035 & 0.035 & 0.005 & 0.005 \\
\hline $50-59$ & 0.042 & 0.046 & 0.006 & 0.007 \\
\hline $60-64$ & 0.053 & 0.055 & 0.009 & 0.010 \\
\hline $65-69$ & 0.049 & 0.050 & 0.014 & 0.014 \\
\hline $70-79$ & 0.072 & 0.076 & 0.029 & 0.030 \\
\hline$\geq 80$ & 0.163 & 0.173 & 0.108 & 0.115 \\
\hline \multicolumn{5}{|c|}{ HES Outpatient consultation } \\
\hline $18-49$ & 0.095 & 0.116 & 0.041 & 0.045 \\
\hline $50-59$ & 0.086 & 0.122 & 0.062 & 0.086 \\
\hline $60-64$ & 0.136 & 0.180 & 0.065 & 0.078 \\
\hline $65-69$ & 0.146 & 0.217 & 0.085 & 0.108 \\
\hline $70-79$ & 0.165 & 0.267 & 0.113 & 0.181 \\
\hline$\geq 80$ & 0.173 & 0.313 & 0.149 & 0.231 \\
\hline \multicolumn{5}{|c|}{ CPRD Ambulatory visits } \\
\hline $18-49$ & 2.816 & 3.168 & 2.186 & 2.360 \\
\hline $50-59$ & 3.334 & 4.175 & 2.466 & 2.907 \\
\hline $60-64$ & 3.733 & 5.081 & 2.598 & 3.115 \\
\hline $65-69$ & 4.089 & 6.009 & 2.774 & 3.610 \\
\hline $70-79$ & 4.534 & 6.959 & 3.413 & 4.767 \\
\hline$\geq 80$ & 4.881 & 7.422 & 3.811 & 5.367 \\
\hline \multicolumn{5}{|c|}{ CPRD Other ambulatory visits } \\
\hline $18-49$ & 0.319 & 0.411 & 0.155 & 0.170 \\
\hline $50-59$ & 0.433 & 0.623 & 0.218 & 0.277 \\
\hline $60-64$ & 0.545 & 0.885 & 0.251 & 0.360 \\
\hline $65-69$ & 0.607 & 1.064 & 0.330 & 0.454 \\
\hline $70-79$ & 0.686 & 1.251 & 0.417 & 0.722 \\
\hline$\geq 80$ & 0.860 & 1.616 & 0.668 & 1.183 \\
\hline \multicolumn{5}{|c|}{ CPRD Prescriptions (all treatments) } \\
\hline $18-49$ & 1.247 & 1.363 & 0.890 & 0.931 \\
\hline $50-59$ & 1.670 & 1.994 & 1.143 & 1.227 \\
\hline $60-64$ & 1.969 & 2.602 & 1.379 & 1.489 \\
\hline $65-69$ & 2.129 & 2.894 & 1.473 & 1.717 \\
\hline $70-79$ & 2.310 & 3.295 & 1.814 & 2.347 \\
\hline$\geq 80$ & 2.405 & 3.743 & 1.844 & 2.575 \\
\hline \multicolumn{5}{|c|}{ CPRD Referrals* } \\
\hline $18-49$ & 0.018 & 0.020 & 0.011 & 0.012 \\
\hline $50-59$ & 0.021 & 0.026 & 0.018 & 0.022 \\
\hline $60-64$ & 0.031 & 0.040 & 0.020 & 0.024 \\
\hline $65-69$ & 0.031 & 0.044 & 0.015 & 0.023 \\
\hline $70-79$ & 0.033 & 0.054 & 0.031 & 0.047 \\
\hline$\geq 80$ & 0.040 & 0.065 & 0.029 & 0.048 \\
\hline \multicolumn{5}{|c|}{ CPRD Sick leave* } \\
\hline $18-49$ & 0.162 & 0.175 & 0.155 & 0.161 \\
\hline $50-59$ & 0.156 & 0.178 & 0.173 & 0.182 \\
\hline
\end{tabular}


Table 1 Continued

\begin{tabular}{|c|c|c|c|c|}
\hline & \multicolumn{2}{|c|}{ IC cohort } & \multicolumn{2}{|c|}{ IC-free cohort } \\
\hline & 90 day & 365 day & 90 day & 365 day \\
\hline $60-64$ & 0.060 & 0.069 & 0.080 & 0.087 \\
\hline $70-79$ & 0.001 & 0.001 & 0.002 & 0.003 \\
\hline$\geq 80$ & 0.000 & 0.000 & 0.000 & 0.000 \\
\hline $18-69$ & 0.000 & 0.000 & 0.000 & 0.000 \\
\hline $70-79$ & 0.001 & 0.001 & 0.001 & 0.001 \\
\hline$\geq 80$ & 0.004 & 0.004 & 0.003 & 0.003 \\
\hline
\end{tabular}

Costs were assigned for HES hospital admission, HES outpatient consultation, CPRD ambulatory Visits, CPRD other ambulatory visits, CPRD prescriptions.

*No costs were assigned for CPRD Referrals, CPRD sick leave, CPRD Nursing home care/admission.

CPRD, Clinical Practice Research Datalink; HES, Hospital Episode Statistics; IC, immunocompromised.

Only events related to $\mathrm{HZ}$ were costed out. Resources related to $\mathrm{HZ}$ complications were considered using ICD-10 Code B020.

No costs were assigned to Referrals, Sick leave or Nursing home care/admission entries in CPRD. Further details, including information on the IC populations included, ICD-10 codes for $\mathrm{HZ}$ and PHN, and unit healthcare costs are provided in the online supplementary material, specifically in online supplementary tables 1 to 4 .

\section{RESULTS}

The CPRD-HES-linked matched IC and IC-free population cohorts $(\mathrm{n}=621588 \mathrm{each})$ included approximately $44 \%$ males and $56 \%$ females with a mean age of approximately 56 years. The age distribution of matched cohorts was: $18-44$ YOA (28.8\%), 45-49 YOA (7.1\%), 50-59 YOA $(17.2 \%), 60-64$ YOA $(9.9 \%), 65-69$ YOA $(9.4 \%), 70-79$ YOA $(16.6 \%)$ and $\geq 80$ YOA $(11.01 \%)$.

The proportion of inpatient hospital admissions by age group for the CPRD-HES-linked matched IC and IC-free cohorts over the time periods of 7 days prior to 90 days post initial HZ onset (Panel A) or 7 days prior to 365 days post initial HZ onset (Panel B) are presented in figure 2. Hospital admissions over the longer follow-up period of 7 days prior to 365 days post initial HZ onset (Panel B) were similar to those of the shorter follow-up period (Panel A) over all age groups. The percentage of $\mathrm{HZ}$ cases hospitalised were higher in IC individuals (eg, in Panel B 2.7\% vs $0.4 \%$ in IC and IC-free individuals aged 18-49 YOA, respectively and $9.5 \%$ vs $7.5 \%$ in IC and IC-free individuals aged $\geq 80 \mathrm{YOA}$, respectively). Multiple HZ-related hospital visits were reported for some individuals. As such, table 1 presents the mean number of healthcare resources used by IC Status, Age Group and Analysis period. The mean number of hospitalizations per HZ case for the 365-day analysis was, 0.035 and 0.005 in IC and IC-free individuals aged 18-49 YOA, respectively and 0.173 and 0.115 in IC and IC-free individuals aged $\geq 80 \mathrm{YOA}$, respectively. A similar pattern of higher healthcare resource utilisation with increasing age and in IC individuals was observed for all resources for which costs were assigned. A similar mean number of sick leave certificates were observed between the IC and the IC-free cohorts with the mean decreasing with

\section{Panel A}

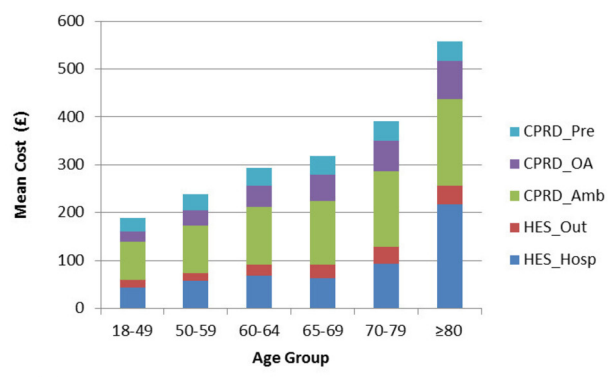

Panel B

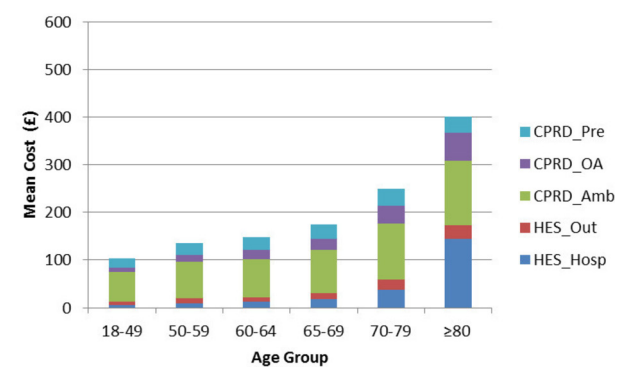

Figure 3 Healthcare costs by HES-linked matched IC (Panel A) and IC-free cohort (Panel B) for the analysis period 7 days prior to 365 days post initial $\mathrm{HZ}$ onset. For $\mathrm{HZ}$ individuals without $\mathrm{PHN}$ : data from 7 days prior to 30 days post $\mathrm{HZ}$ onset included. For $\mathrm{HZ}$ individuals with $\mathrm{PHN}$ : data from 7 days prior until 365 days after $\mathrm{HZ}$ onset. $£, 2014$ UK pound sterling; CPRD, Clinical Practice Research Datalink; CPRD_Amb, CPRD Ambulatory Visits; CPRD_OA, CPRD Other Ambulatory Visits; CPRD_Pre, CPRD Prescriptions; HES, Hospital Episode Statistics; HES_Hosp, HES Hospital admission; HES_Out, HES Outpatient consultation; HZ, herpes zoster; IC, immunocompromised; PHN, postherpetic neuralgia. 
Table 2 Mean cost ( () of healthcare resource utilisation by IC status, age group and analysis period ${ }^{\star}$

\begin{tabular}{|c|c|c|c|c|c|}
\hline \multirow[b]{3}{*}{ Age groups (YOA) } & \multirow[b]{3}{*}{ Statistic } & \multicolumn{4}{|c|}{ Mean cost (£) } \\
\hline & & \multicolumn{2}{|c|}{ IC cohort } & \multicolumn{2}{|c|}{ IC-free cohort } \\
\hline & & 90 day & 365 day & 90 day & 365 day \\
\hline \multirow[t]{2}{*}{$18-49$} & Mean & 173.3 & 189.3 & 98.2 & 103.8 \\
\hline & Median, SE & $86.1,6.03$ & $86.9,6.81$ & $59.6,3.06$ & $62.0,3.35$ \\
\hline \multirow[t]{2}{*}{$50-59$} & Mean & 199.0 & 237.8 & 118.9 & 135.3 \\
\hline & Median, SE & $106.6,6.37$ & $108.8,9.05$ & $74.6,3.72$ & $74.8,4.68$ \\
\hline \multirow[t]{2}{*}{$60-64$} & Mean & 236.2 & 294.2 & 126.8 & 147.7 \\
\hline & Median, SE & $120.2,9.39$ & $124.1,12.01$ & $78.9,4.48$ & $80.9,5.81$ \\
\hline \multirow[t]{2}{*}{$65-69$} & Mean & 241.6 & 317.4 & 145.5 & 174.4 \\
\hline & Median, SE & $132.2,8.52$ & $140.0,11.25$ & $87.9,4.64$ & $90.7,5.97$ \\
\hline \multirow[t]{2}{*}{ 70-79 } & Mean & 289.6 & 391.7 & 189.8 & 248.6 \\
\hline & Median, SE & $154.2,7.21$ & $163.9,10.11$ & $108.8,4.97$ & $113.6,6.88$ \\
\hline \multirow[t]{2}{*}{$\geq 80$} & Mean & 427.0 & 557.1 & 319.7 & 401.0 \\
\hline & Median, SE & $176.2,13.12$ & $188.6,17.05$ & $143.0,11.20$ & $154.0,13.63$ \\
\hline
\end{tabular}

*Post initial HZ onset.

£, 2014 UK pound sterling; HZ, herpes zoster; IC, immunocompromised; SE, Standard Error; YOA, years of age.

age. Nursing home care/admissions were only recorded for individuals aged $\geq 70 \mathrm{YOA}$ in CPRD.

Figure 3 and table 2 present the overall healthcare costs by CPRD-HES-linked matched IC cohort and age group for the analysis period 7 days prior to 365 days post initial $\mathrm{HZ}$ onset. The costs increase with age and are consistently higher in the IC cohort compared with the IC-free cohort. Although the absolute cost difference between IC and IC-free individuals increases with age from $£ 85.5$ in individuals aged $18-49$ YOA to $£ 156.1$ in individuals aged $\geq 80$ YOA the relative difference is higher in younger individuals (ie, $75.8 \%-99.2 \%$ in $<70 \mathrm{YOA}$ ) compared with older individuals (ie, $38.9 \%-57.6 \%$ in $\geq 70 \mathrm{YOA}$ ). It is also noteworthy that the means are consistently higher than medians, and as is common for healthcare cost data, the distribution is skewed to the right. See online supplementary table 5 and online supplementary figures 1 and 2 provide additional data on healthcare Costs for the analysis period 7 days prior to 90 days post initial $\mathrm{HZ}$ onset.

Figure 4 presents the overall healthcare costs by each IC condition in the CPRD-HES-linked matched IC and IC-free cohort by age group for the analysis period 7 days prior to 365 days post initial HZ onset. For all IC conditions, the costs were higher than those for the IC-free group, in particular for the hematopoietic stem cell transplantation (HSCT), haematological malignancies and solid organ transplantations (SOT) conditions. In general, there was a similar trend of increasing costs with increasing age-groups. A few outliers were observed due to small sample sizes. For example, only 3 and 8 individuals aged $\geq 70$ YOA were included in the HIV and HSCT groups, respectively. Similarly, in total only 207 and 271 individuals with autoimmune thyroiditis and SOT were included, respectively.
Table 3 presents the mean healthcare costs by IC status and HZ complication status. The mean healthcare costs were approximately 4 to 5 times higher for individuals with PHN for the analysis period 7 days prior to 365 days compared with individuals with HZ only. Similarly, mean healthcare costs were approximately 2 to 4 times higher for individuals with $\mathrm{HZ}$ complications compared with individuals with $\mathrm{HZ}$ only.

Online supplementary table 6 presents the non-HZ related hospital inpatient stay for the period 7 days to 365 days post initial-HZ onset. The mean number of non-HZ related hospitalisations were consistently higher in IC patients compared with and IC-free patients and increased with age.

\section{DISCUSSION}

In this study, we presented the healthcare resource utilisation and costs associated with HZ in both IC and IC-free populations using large electronic health record databases in the UK. An important feature of this study was that the design enabled the calculation of IC condition prevalence rates, $\mathrm{HZ}$ incidence rates and occurrence of HZ-related healthcare utilisation and costs at individual level in the same predefined population(s), see Yanni et al for further detail on epidemiological outcomes. ${ }^{7}$ In this study, every effort was made to include only resources directly related to HZ. For example, only hospitalised patients were included who had an ICD-10 HZ diagnosis identified in the HES database. Similarly, only medications potentially related to HZ treatment were included (see online supplementary tables 2 and 4). HZ-related mean treatment costs per patient were higher in IC individuals (£189 vs £104 in IC and IC-free 
Panel A

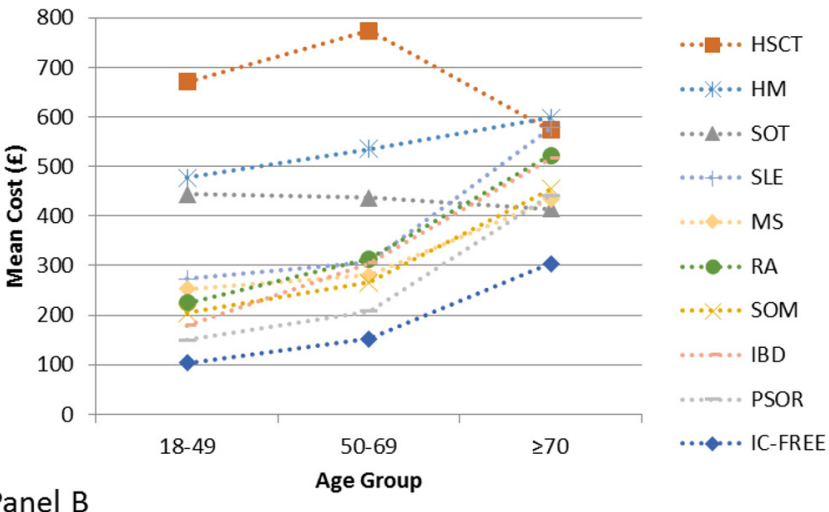

Panel B

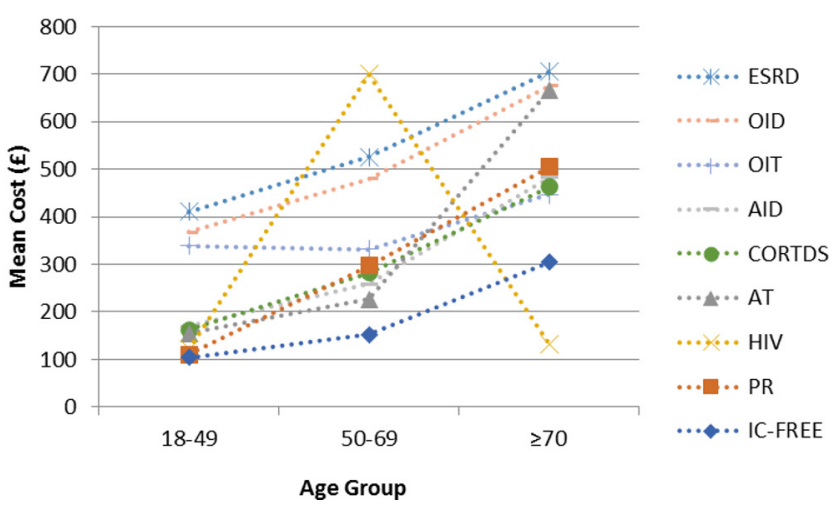

Figure 4 Healthcare costs for each IC condition in the HES-linked matched IC and IC-free cohort by age group for the analysis period 7 days prior to 365 days post initial $\mathrm{HZ}$ onset. For $\mathrm{HZ}$ individuals without PHN: data from 7 days prior to 30 days post $\mathrm{HZ}$ onset included. For $\mathrm{HZ}$ individuals with PHN: data from 7 days prior until 365 days after $\mathrm{HZ}$ onset. £, 2014 UK pound sterling; AID, autoimmune diseases; AT, autoimmune thyroiditis; CORTDS, corticosteroid exposure; ESRD, end-stage renal disease; HES, Hospital Episode Statistics; HIV, human immunodeficiency virus; HM, haematological malignancies; HSCT, hematopoietic stem cell transplantation; $\mathrm{HZ}$, herpes zoster; IBD, inflammatory bowel syndrome; IC, immunocompromised; MS, multiple sclerosis; OID, other immunodeficiency; OIT, other immunosuppressive therapy; PHN, postherpetic neuralgia; PR, polymyalgia rheumatica; PSOR, psoriasis; RA, rheumatoid arthritis; SLE, systemic lupus erythematosus; SOM, solid organ malignancies; SOT, solid organ transplantations.

individuals aged 18-49 YOA, respectively increasing to $£ 557$ vs $£ 401$ in IC and IC-free individuals aged $\geq 80$ YOA, respectively).

Previous studies of healthcare costs of $\mathrm{HZ}$ in the UK, included a small study, which estimated the mean healthcare costs per $\mathrm{HZ}$ patient, from an National Health Services perspective, of $£ 85.6$ and $£ 400.9$ in individuals aged $<65$ YOA and $\geq 65$ YOA, respectively. ${ }^{12}$ A later UK study that used the HES and the health improvement network databases, estimated the mean cost of treating a $\mathrm{HZ}$ patient to be $£ 65.5$ in the first month of diagnosis, with patients aged $\geq 70$ YOA having a mean cost of $£ 83$ in the first month and $£ 15.80$ in months 2 and 3 . $^{13}$ The costs of treating individuals with $\mathrm{PHN}$ were much higher, that is, mean cost per patient was estimated to be $£ 921$ in all individuals and $£ 909.60$ in individuals aged $\geq 70$ YOA. $^{13}$ Another study evaluated mean healthcare costs (excluding hospitalisation costs) to be $£ 75.63$ per $\mathrm{HZ}$ patient with mean direct costs for treating PHN episodes (PHN pain occurring or persisting for 3 months) of $£ 340.04 .{ }^{14}$ These values augmented with hospitalisation costs were used as inputs in a cost-effectiveness model evaluating a $\mathrm{HZ}$ vaccine using the population of England and Wales. ${ }^{3}$ The costs estimated by van Hoek $e t$ $a l$ are consistent with the values estimated in our study for IC-free individuals by age group. ${ }^{3}$

In a previous study, mean prescription costs per $\mathrm{HZ}$ patient were reported to be $£ 40.52 .{ }^{14}$ In our study, the mean prescription costs per HZ patient ranged from $£ 19.7$ to $£ 40.8$ depending on the age group, IC status and analysis period included. Our study aimed to include only medications considered to be directly related to $\mathrm{HZ}$; that is, excluded medications that may be linked to IC conditions (eg, aspirin, analgesic creams as they could be used primarily to reduce pain from other conditions). This restriction and the introduction of generic versions of medications such as acyclovir, gabapentin (and derivatives of gabapentin) which resulted in lower prices, contributed to the reduced overall medication costs reported in this study.

Many studies on HCRU and costs include a number of days prior to diagnosis, for example, 14 or 21 days, as there may be a delay in diagnosis and HCRU may be used prior to diagnosis. ${ }^{615}$ In this analysis, costs of $\mathrm{HZ}$ only cases were assessed during the period 7 days prior to 30 days post $\mathrm{HZ}$ onset, although it is recognised that $\mathrm{HZ}$ episodes can last for longer. The costs of PHN were analysed over two time-periods, ie, (1) 7 days prior to 90 days post $\mathrm{HZ}$ onset and (2) 7 days prior to 365 days post $\mathrm{HZ}$ onset. The rationale for the time periods studied was that using analysis period 1 alone could lead to an underestimation of PHN costs whereas using analysis period 2 only could overestimate these costs. The most frequently used definition of $\mathrm{PHN}$ is: pain persisting or appearing at least 90 days following rash onset. The median duration of PHN has been reported to be 10.3 and 12.9 months in individuals aged $\leq 69$ and $\geq 70$ YOA respectively, ${ }^{16}$ and is likely to be longer in individuals who are immunocompromised. ${ }^{5}$

The healthcare costs associated with PHN and complications were higher than those for individuals with $\mathrm{HZ}$ only. However, as reported elsewhere, when considering the overall cost of disease at a population level, the overall healthcare-associated cost is higher for $\mathrm{HZ}$ only. ${ }^{17}$ This is primarily a result of the higher incidence rates of $\mathrm{HZ}$ only.

Few studies have investigated healthcare resource utilisation and costs in IC individuals. Schroder et al carried out a study using the German Pharmacoepidemiological Research Database, which consists of claims data from four statutory health insurances. ${ }^{18}$ They reported that during the quarter of the $\mathrm{HZ}$ diagnosis or during the two following quarters, $10 \%$ of all $\mathrm{HZ}$ patients with an IC condition were hospitalised (with a HZ diagnosis), whereas among IC-free HZ patients, $4.2 \%$ were hospitalised. White et al reported that in their study using the US Market Scan Research 
Table 3 Mean cost $(£)$ of healthcare resource utilisation by IC status, age group, analysis period and $\mathrm{HZ}$ complication status

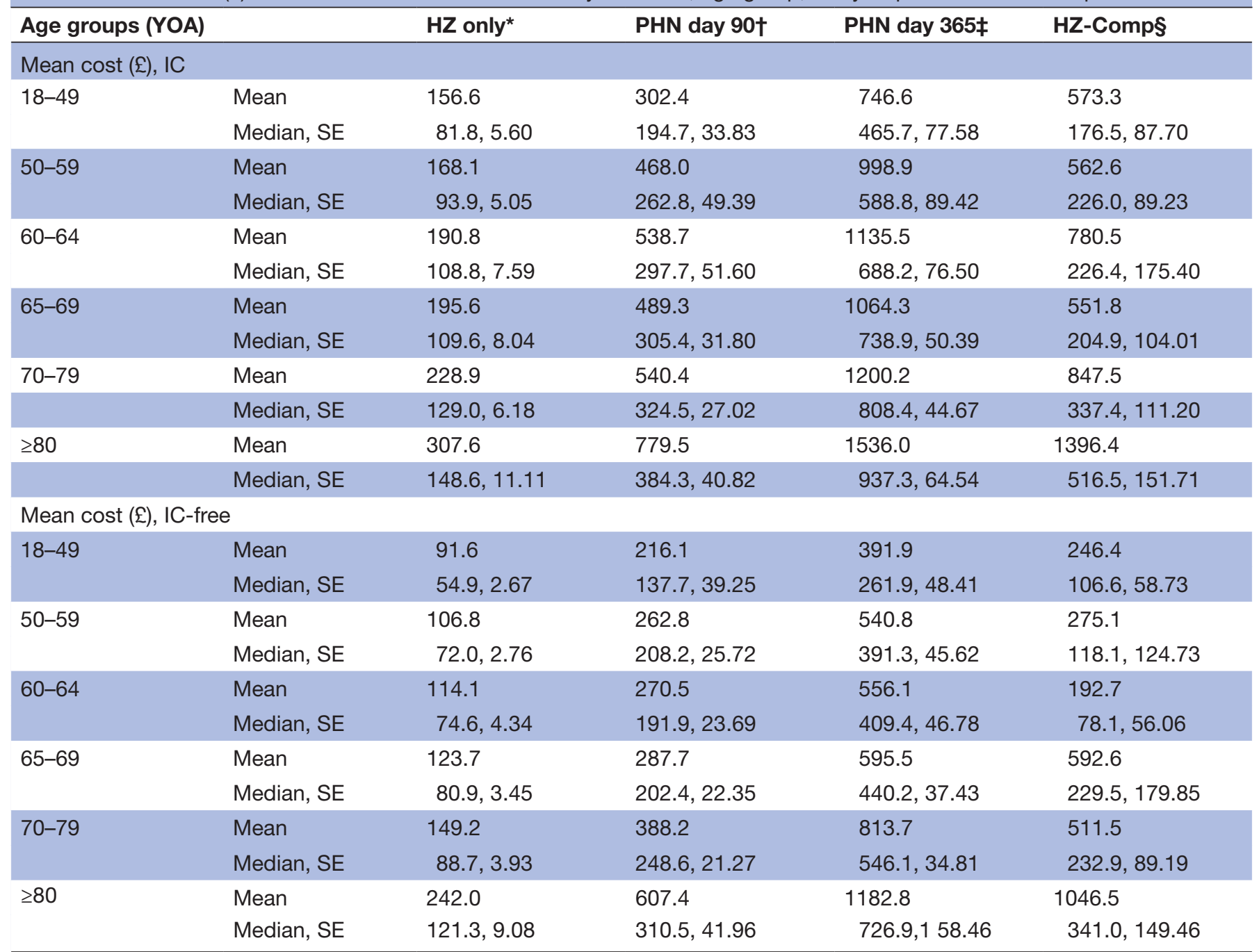

*Individuals with $\mathrm{HZ}$ only (ie, without PHN and complications): includes only costs 7 days prior to 30 days post initial $\mathrm{HZ}$ onset. †Individuals with $\mathrm{HZ}$ and $\mathrm{PHN}$ : includes only costs 7 days prior to 90 days post initial $\mathrm{HZ}$ onset. fIndividuals with $\mathrm{HZ}$ and PHN: includes costs 7 days prior to 365 days post initial $\mathrm{HZ}$ onset.

§Individuals with $\mathrm{HZ}$ and complications but no PHN: includes only costs 7 days prior to 30 days post initial $\mathrm{HZ}$ onset.

£: 2014 UK pound sterling; HZ,herpes zoster; HZ-Comp, HZ and complications with no PHN; IC, immunocompromised; PHN, postherpetic neuralgia; SE, Standard Error; YOA, years of age.

Database, direct medical costs were nearly twice as high in IC patients compared with IC-free patients. ${ }^{19} \mathrm{Li}$ et al carried out a study using the US Truven Health MarketScan Commercial and Medicare Supplemental Insurance databases. ${ }^{15}$ They concluded that patients with the studied IC conditions (ie, HIV, SOT, bone marrow or stem cell transplant and cancer) had significantly higher healthcare utilisation and cost when developing $\mathrm{HZ}$ than their comparable matches without HZ. Insurance databases include not only the healthcare resource utilisation but also costs. In the CPRD and HES Databases only the resource utilisation is captured. As such the overall costs need to be calculated by assigning unit costs to the resource utilisation. There are advantages however of using the CPRD and HES in that the databases offer more diversity than might be observed using insurance databases, the latter of which may be somewhat limited by bias associated with factors such as age, race and income. A strength of the CPRD database is that it is considered to be broadly representative of the characteristics of patients and GP practices in the UK. ${ }^{20} 21$

This study has several limitations. Diagnoses were derived from administrative codes, which are recognised to be subject to miscoding or under-coding and are not validated against medical charts. ${ }^{22}$ Increasing healthcare resource utilisation and cost is likely to be related to increased severity of IC conditions. In a study, Schroder et al categorised individuals as low IC and high IC. ${ }^{18}$ However, insufficient details are recorded in the CPRD and HES databases to allow adequate definition of patients' severity of immunosuppression for example, laboratory parameters, immunosuppressive medication details such as chemotherapy. In addition, many IC individuals had prescriptions that included more 
than one immunosuppressing medicine. In this study we selected 16 IC conditions in our definition of an IC population but perhaps other researchers would select different IC conditions. As such our study is exploratory in nature and was not intended to be definitive.

\section{CONCLUSION}

Immunosuppression is known to be associated with an increased risk of $\mathrm{HZ}$ in the $\mathrm{UK} .^{27}$ In this descriptive analysis, involving a large representative national data source, the results suggest that individuals with IC conditions were associated with higher $\mathrm{HZ}$ related healthcare utilisation and costs than IC-free individuals. ${ }^{6715}$ The results from this study could be used in economic analyses to evaluate the value of vaccination in reducing the burden of $\mathrm{HZ}$ in these populations.

Acknowledgements The authors would like to thank Emmanuelle Espié, Emad Yanni, Morgane Guinnec, François Haguinet for their contribution to the study. They would also like to thank the Business \& Decision Life Sciences platform for editorial assistance and coordination, on behalf of GSK. Gregory Collet coordinated manuscript development and editorial support. Kathleen Daly provided editing support. This study is based in part on data from the Clinical Practice Research Datalink obtained under licence from the UK Medicines and Healthcare products Regulatory Agency. However, the interpretation and conclusions contained in this report are those of the authors alone. Part of the data have been previously published as an abstract (Curran al., 2017) and presented at ISPOR-EU (2017) International Society for Pharmacoeconomics and Outcomes Research - 20th Annual European Congress.

Contributors VB, AEG, YEH, GF, MH and DC participated in the conception and design of the study. VB, AEG, YEH, GF and MH participated in the collection or generation of the study data. VB, AEG and YEH performed the study. AEG, YEH, MH and $D C$ contributed to the material. VB, AEG, YEH, GF, MH and DC were involved in the analysis or interpretation of the data. All named authors provided substantial intellectual and scientific input during the manuscript development, critically reviewing the content, revising the manuscript and giving final approval before submission. The work described was carried out in accordance with the ICMJE recommendations for conducting, reporting, editing and publishing scholarly work in medical journals. All authors had full access to the data and gave final approval before submission. The corresponding author was responsible for submission of the publication.

Funding GlaxoSmithKline Biologicals SA was the funding source and was involved in all study (GSK study identifier: e-track number: 201615) activities and overall data management (collection, analysis and interpretation). GlaxoSmithKline Biologicals SA also funded all costs associated with the development and the publishing of the present manuscript.

Competing interests VB, MH and DC are employees of the GSK group of companies. DC and MH hold shares in the GSK group of companies. AEG and YEH have nothing to disclose. GF was employed by the GSK group of companies between 2012 and Feb 2015, during which the study was designed and implemented. Later, as an employee of P-95 epidemiology and pharmacovigilance, GF provided contracted consultancy services to the GSK group of companies for this and other GSK-sponsored studies. P-95 provides contracted services to the GSK group of companies, beyond the scope of this study.

Patient consent for publication Not required.

Ethics approval Approval was obtained from the Clinical Practice Research Datalink Independent Scientific Advisory Committee (14_222R).

Provenance and peer review Not commissioned; externally peer reviewed.

Data sharing statement All data used in this study are presented in the manuscript, references to the original material are provided. Please contact the corresponding author shall you require any additional information.
Author note AEG and YEH were consultant for GSK, Wavre, Belgium at the time of the study. AEG is currently at F. Hoffmann-La Roche Ltd., Basel, Switzerland and YEH is currently at Accord Research s.r.o., Prague, Czech Republic.

Open access This is an open access article distributed in accordance with the Creative Commons Attribution Non Commercial (CC BY-NC 4.0) license, which permits others to distribute, remix, adapt, build upon this work non-commercially, and license their derivative works on different terms, provided the original work is properly cited, appropriate credit is given, any changes made indicated, and the use is non-commercial. See: http://creativecommons.org/licenses/by-nc/4.0/.

\section{REFERENCES}

1. Oxman MN. Zoster vaccine: current status and future prospects. Clin Infect Dis 2010;51:197-213.

2. Forbes HJ, Bhaskaran K, Thomas SL, et al. Quantification of risk factors for herpes zoster: population based case-control study. BMJ 2014;348:g2911.

3. van Hoek AJ, Gay N, Melegaro A, et al. Estimating the costeffectiveness of vaccination against herpes zoster in England and Wales. Vaccine 2009;27:1454-67.

4. Coplan PM, Schmader K, Nikas A, et al. Development of a measure of the burden of pain due to herpes zoster and postherpetic neuralgia for prevention trials: adaptation of the brief pain inventory. $J$ Pain 2004;5:344-56.

5. Drolet M, Brisson M, Schmader K, et al. Predictors of postherpetic neuralgia among patients with herpes zoster: a prospective study. $J$ Pain 2010;11:1211-21.

6. Yawn BP, Itzler RF, Wollan PC, et al. Health care utilization and cost burden of herpes zoster in a community population. Mayo Clin Proc 2009;84:787-94.

7. Yanni EA, Ferreira G, Guennec M, et al. Burden of herpes zoster in 16 selected immunocompromised populations in England: a cohort study in the Clinical Practice Research Datalink 2000-2012. BMJ Open 2018;8:e020528.

8. Brazauskas R, Logan BR. Observational studies: matching or regression? Biol Blood Marrow Transplant 2016;22:557-63.

9. International Society for Pharmacoepidemiology. Guidelines for Good Pharmacoepidemiology Practices (GPP). 2015 https://www. pharmacoepi.org/resources/guidelines_08027.cfm

10. Curtis LPSSRU. Unit costs of health \& social care 2014. 2014 http:// www.pssru.ac.uk/project-pages/unit-costs/2014/

11. National Tariff Payment System. Annex 5A national prices 2015. 2014 https://www.gov.uk/government/publications/national-tariffpayment-system-2014-to-2015

12. Scott FT, Johnson RW, Leedham-Green M, et al. The burden of Herpes Zoster: a prospective population based study. Vaccine 2006;24:1308-14.

13. Taieb V, Schwarzbard J, Butt T, et al. The Epidemiological and Cost Burden of Herpes Zoster $(\mathrm{Hz})$ and Post-Herpetic Neuralgia (Phn) in the Uk. Value Health 2015;18:A589.

14. Gauthier A, Breuer J, Carrington D, et al. Epidemiology and cost of herpes zoster and post-herpetic neuralgia in the United Kingdom. Epidemiol Infect 2009;137:38-47.

15. Li Q, Chen SY, Burstin SJ, et al. Cost of Herpes Zoster in Patients With Selected Immune-Compromised Conditions in the United States. Open Forum Infect Dis 2016;3:ofw067.

16. Moore L, Remy V, Martin M, et al. A health economic model for evaluating a vaccine for the prevention of herpes zoster and postherpetic neuralgia in the UK. Cost Eff Resour Alloc 2010;8:7.

17. Gater A, Uhart M, McCool R, et al. The humanistic, economic and societal burden of herpes zoster in Europe: a critical review. BMC Public Health 2015;15:193.

18. Schröder C, Enders D, Schink T, et al. Incidence of herpes zoster amongst adults varies by severity of immunosuppression. $J$ Infect 2017;75:207-15.

19. White RR, Lenhart G, Singhal PK, et al. Incremental 1-year medical resource utilization and costs for patients with herpes zoster from a set of US health plans. Pharmacoeconomics 2009;27:781-92.

20. Campbell J, Dedman DJ, Eaton SC, et al. Is the CPRD GOLD population comparable to the U.K. population? Pharmacoepidemiol Drug Saf 2013;22:280.

21. Herrett E, Gallagher AM, Bhaskaran K, et al. Data resource profile: Clinical Practice Research Datalink (CPRD). Int J Epidemiol 2015;44:827-36.

22. Pierik JG, Gumbs PD, Fortanier SA, et al. Epidemiological characteristics and societal burden of varicella zoster virus in the Netherlands. BMC Infect Dis 2012;12:110. 